\title{
Green Supplier Selection Based on Dombi Prioritized Bonferroni Mean Operator with Single-Valued Triangular Neutrosophic Sets
}

\author{
Jianping Fan, Xuefei Jia, Meiqin $\mathrm{Wu}^{*}$ \\ School of Economics and Management, Shanxi University, Taiyuan 030006, China
}

\author{
ARTICLE INFO \\ Article History \\ Received 02 Jul 2019 \\ Accepted 15 Sep 2019 \\ Keywords \\ Single-valued triangular \\ Neutrosophic sets \\ Dombi operations \\ Prioritized operator \\ Bonferroni mean operator \\ Dombi prioritized normalized \\ Bonferroni mean operator
}

\begin{abstract}
The choice of green suppliers involves a large amount of inaccurate, incomplete, and inconsistent information, and the singlevalued triangular Neutrosophic number that is an extension of the single-valued Neutrosophic number can effectively handle such problems. Considering the advantages of the single-valued triangular Neutrosophic number, this paper proposes a new aggregate operator to solve the problem of multi-criteria decision making. The new aggregate operator takes into account the priority relationship and the interrelationship between the criteria. To make the new aggregate operator more flexible, this paper introduces the Dombi operations. This paper combines the Dombi operations with the prioritized average operator and the Bonferroni mean operator to propose the single-valued triangular Neutrosophic Dombi prioritized normalized Bonferroni mean (SVTNDPNBM) operator. Finally, the SVTNDPNBM operator is applied to the problem of the green supplier selection, which proves its feasibility and stability.
\end{abstract}

(C) 2019 The Authors. Published by Atlantis Press SARL.

This is an open access article distributed under the CC BY-NC 4.0 license (http://creativecommons.org/licenses/by-nc/4.0/).

\section{INTRODUCTION}

In recent years, consumers, business operators, and governments are paying more and more attention to green development and environmental performance issues, with the rapid consumption of resources and serious environmental pollution. In this context, companies that want to have competitive advantage in the market cannot ignore the environmental factors in supplier selection. Choosing the appropriate green supplier can improve market competitive advantage and environmental performance. But choosing the right green supplier requires consideration of criteria such as product quality, cost, service capability, green image, and innovation capability. In essence, the process of choosing the best green supplier is a multi-criteria decision-making (MCDM) problem.

After Zadeh [1] proposed the concept of fuzzy sets (FS), FS were widely used. But the FS represent the uncertainty of decision information only by using the membership degree. Atanassov [2] proposed intuitionistic fuzzy sets (IFS) by introducing nonmembership degree, which can effectively deal with the problems that FS cannot handle. Gargov et al. [3] and Atanassov [4] extended the IFS to interval numbers, and proposed interval IFS (IIFS). Torra [5] defined hesitant fuzzy sets (HFS), which can deal with the uncertainty caused by the decision maker's hesitation well. Qian et al. [6] and Zhu et al. [7] defined generalized HFS and double HFS, respectively. However, sometimes the sum of membership degree and non-membership degree is greater than one. Yager [8] proposed Pythagorean fuzzy sets (PFS) to solve this problem, allowing the sum of membership degree and non-membership degree to be greater than one, while satisfying the sum of the squares of the membership degree and the non-membership degree is less than or equal to one. Although FSs theory has been extensively studied and expanded, FS and their extension sets cannot handle discontinuous and inconsistent information. The emergence of the Neutrosophic sets (NS) just makes up for this deficiency. Smarandache [9] proposed the concept of the NS, which uses the truth-membership function, the indeterminacy-membership function and the falsitymembership function to depict the fuzzy information, and these are independent of each other.

Although the NS expand the expression of uncertain information, it is very inconvenient in practical applications. To simplify the NS, Ye [10] proposed the concept of simplified Neutrosophic sets (SNS), and pointed out SNS contain single-valued Neutrosophic sets (SVNS) and interval Neutrosophic sets (INS). Ye [11] proposed aggregation operators and cosine similarity measurement of SNS. Peng, Wang et al. [12] improved the operations of SNS in the literature [11]. Biswas et al. [13] combined triangular fuzzy numbers with SVNS, and proposed single-valued triangular Neutrosophic sets (SVTNS). Wang et al. [14] considered the conflicting criteria, extended the original VIKOR model to SVTNS, and introduced the specific steps to apply the method. 
The aggregation operators present the powerful tool for handling MCDM problem. Dombi [15] proposed Dombi operations, including T-norm and T-conorm, which show the advantage of good flexibility with the operation of parameters. Recently, several authors defined Dombi operations in IFS [16], SVNS [17], HFS [18]. Yager [19] proposed prioritized average (PA) operator by considering the priority relationship between the criteria. The PA operator has been widely used in a variety of fuzzy numbers, such as HFS [20], uncertain linguistic sets [21], interval-valued hesitant fuzzy [22], INS [23]. In order to solve the relationship between the criteria, Bonferroni [24] proposed the Bonferroni mean (BM) aggregation operator. Yager et al. [25] defined weighted Bonferroni mean (WBM) operator based on the BM operator, but WBM has the disadvantage of non-reducibility. In order to overcome this deficiency, Zhou and $\mathrm{He}$ [26] defined the normalized weighted Bonferroni mean (NWBM) operator based on the WBM operator. Tian et al. [27] proposed the gray linguistic BM operator to address the situations where the criterion values take the form of gray linguistic numbers and the criterion weights are known. Each operator has its own unique advantages. Liu et al. [16] proposed some Dombi BM operators in the IFS environment. Khan et al. [28] proposed Dombi power BM operators in the INS environment. Nie et al. [29] proposed partitioned NWBM operator based on Shapley fuzzy measures, in the PFS environment. Considering the advantages of the Hamy mean operator, Li et al. [30] proposed Dombi Hamy mean operators in the IFS environment, and $\mathrm{Wu}$ et al. [31] proposed Dombi Hamy mean operators in the IIFS environment. Yager et al. [32] applied the Dombi operators to Picture fuzzy, and Zhang et al. [33] proposed Picture fuzzy Dombi Heronian mean operators. Wei and Zhang [34] introduced Bonferroni power operators into SVNS environment. Wang et al. [35] combined Frank operational laws to proposed Frank prioritized BM operator.

Choosing the right green supplier can eliminate some environmental impact and improve environmental performance. So far, the research of green supplier selection has achieved certain results. Noci [36] pointed out for the first time that measuring the environmental performance of green suppliers includes quantitative and qualitative indicators, and proposed a method of selecting green suppliers from the environmental perspective. Lee et al. [37] used Delphi method to distinguish traditional suppliers from green suppliers and proposed a fuzzy extended analytic hierarchy process model to evaluate green suppliers. Gao et al. [38] used intuitionistic fuzzy numbers for green supplier selection when criteria weights are unknown. Due to the complexity of the environment, the information available for evaluation selection is increasingly uncertain. Liang et al. [39] proposed the single-valued trapezoidal Neutrosophic preference relations as a strategy for tackling green supplier selection problems. Qin et al. [40] considered that decision makers are not entirely reasonable in making decisions, and extend the TODIM technique to solve MCDM problems, then proposed a new method for select the optimal green supplier in the interval type2 FS environment. Yazdani et al. [41] comprehensively considered the evaluation criteria of traditional suppliers and green suppliers, and sorted green suppliers based on the quality function deployment model. Li et al. [42] demonstrated the advantages of probability HFS in decision making process, and combined probability HFS and the extended qualitative flexible multiple method to solve the problem of green supplier selection. Ji et al. [43] conducted a study on green supplier selection in the context of the single-valued Neutrosophic linguistic sets.
According to the existing literature, nobody proposed the Dombi operations of SVTNS, and nobody combined the PA operator with the BM operator for the SVTNS environment. Therefore, it is necessary to propose SVTNDPNDM operator. The SVTNDPNBM operator has some flexibility, and simultaneously considers the priority relationship and interaction between the criteria by integrating the Dombi operations, the PA operator and NWBM operator. In this paper, the SVTNS are used to represent the evaluation value corresponding to different green suppliers, which can represent more uncertain information. The selection of the PA operator can take into account the priority relationship between the criteria. The selection of the BM operator can take into account the interrelationship between the criteria, and introduce the Dombi operations for the flexibility of the operation.

This paper firstly defines the Dombi operations in the SVTNS environment. Then, based on the Dombi operations, we combine the PA operator and BM operator, and propose the single-valued triangular Neutrosophic Dombi Bonferroni mean (SVTNDBM) operator and the single-valued triangular Neutrosophic Dombi prioritized normalized Bonferroni mean (SVTNDPNBM) operator. Finally, based on the proposed new operator, a model is established to solve MCDM problem.

The rest of the paper is structured as follows: In the second section, the related concepts of the SVTNS, Dombi operations, PA operator and BM operator are introduced in detail. The third section proposes the new operator of SVTNS. The forth section builds a model for selecting suitable green suppliers. The fifth section gives a numerical example to prove the feasibility and adaptability of the proposed method. The last part is the conclusion.

\section{PRELIMINARIES}

This section introduces some concepts about the SVTNS, Dombi operations, PA operator, and BM operator. These will be used in later papers.

\subsection{Single-Valued Triangular Neutrosophic Sets}

The SVNS is a good representation of uncertain, incomplete, and inconsistent information in the real world, but decision makers often use fuzzy numbers rather than precise numbers to represent membership function. Biswas et al. [13] defined the SVTNS by combining the triangular fuzzy number and the SVNS.

Definition 1. [13] Let $X$ be a finite set of points (objects), let $x$ denote a generic element in $X$, and $E[0,1]$ be the set of all triangular fuzzy numbers on $[0,1]$. The SVTNS $A$ in $X$ is characterized by a truth-membership function $T_{A}(x)$, an indeterminacy- membership function $I_{A}(x)$ and a falsity-membership function $F_{A}(x)$. Then, the SVTNS $A$ can be depicted as:

$$
A=\left\{\left\langle x, T_{A}(x), I_{A}(x), F_{A}(x)\right\rangle \mid x \in X\right\}
$$

where, $T_{A}(x): X \rightarrow E[0,1], I_{A}(x): X \rightarrow E[0,1]$ and $F_{A}(x): X \rightarrow E[0,1] . T_{A}(x), I_{A}(x), F_{A}(x)$ can be expressed as follows: $T_{A}(x)=\left(T_{A}^{1}(x), T_{A}^{2}(x), T_{A}^{3}(x)\right), I_{A}(x)=\left(I_{A}^{1}(x), I_{A}^{2}(x), I_{A}^{3}(x)\right)$ and $F_{A}(x)=\left(F_{A}^{1}(x), F_{A}^{2}(x), F_{A}^{3}(x)\right)$, for every $x \in X$, satisfy $0 \leq T_{A}^{3}(x)+I_{A}^{3}(x)+F_{A}^{3}(x) \leq 3$. 
For convenience, we consider $A=\langle(a, b, c),(e, f, g),(r, s, t)\rangle$ as SVTN number, where $\left(T_{A}^{1}(x), T_{A}^{2}(x), T_{A}^{3}(x)\right)=(a, b, c)$, $\left(I_{A}^{1}(x), I_{A}^{2}(x), I_{A}^{3}(x)\right)=(e, f, g)$ and $\left(F_{A}^{1}(x), F_{A}^{2}(x), F_{A}^{3}(x)\right)=$ $(r, s, t)$.

Definition 2. [13] Let

$A_{1}=\left\langle\left(a_{1}, b_{1}, c_{1}\right),\left(e_{1}, f_{1}, g_{1}\right),\left(r_{1}, s_{1}, t_{1}\right)\right\rangle, \quad A_{2}=$ $\left\langle\left(a_{2}, b_{2}, c_{2}\right),\left(e_{2}, f_{2}, g_{2}\right),\left(r_{2}, s_{2}, t_{2}\right)\right\rangle$ be two SVTN numbers, the rules of operations can be defined as follows:

1. $A_{1} \oplus A_{2}=\left\langle\begin{array}{l}\left(a_{1}+a_{2}-a_{1} a_{2}, b_{1}+b_{2}-b_{1} b_{2}, c_{1}+c_{2}-c_{1} c_{2}\right), \\ \left(e_{1} e_{2}, f_{1} f_{2}, g_{1} g_{2}\right),\left(r_{1} r_{2}, s_{1} s_{2}, t_{1} t_{2}\right)\end{array}\right\rangle$

2. $A_{1} \otimes A_{2}=\left\langle\begin{array}{l}\left(a_{1} a_{2}, b_{1} b_{2}, c_{1} c_{2}\right), \\ \left(e_{1}+e_{2}-e_{1} e_{2}, f_{1}+f_{2}-f_{1} f_{2}, g_{1}+g_{2}-g_{1} g_{2}\right), \\ \left(r_{1}+r_{2}-r_{1} r_{2}, s_{1}+s_{2}-s_{1} s_{2}, t_{1}+t_{2}-t_{1} t_{2}\right)\end{array}\right\rangle$


$\lambda>0$

$\left(a_{1}^{\lambda}, b_{1}^{\lambda}, c_{1}^{\lambda}\right)$

4. $A_{1}^{\lambda}=\left\langle\begin{array}{l}\left.\left(1-\left(1-e_{1}\right)^{\lambda}, 1-\left(1-f_{1}\right)^{\lambda}, 1-\left(1-g_{1}\right)^{\lambda}\right),\right\rangle, \\ \left(1-\left(1-r_{1}\right)^{\lambda}, 1-\left(1-s_{1}\right)^{\lambda}, 1-\left(1-t_{1}\right)^{\lambda}\right)\end{array}\right.$, $\lambda>0$

The above operations satisfy the following properties:

1. $A_{1} \oplus A_{2}=A_{2} \oplus A_{1} ; A_{1} \otimes A_{2}=A_{2} \otimes A_{1}$

2. $\lambda\left(A_{1} \oplus A_{2}\right)=\lambda A_{1} \oplus \lambda A_{2}$; $\left(A_{1} \otimes A_{2}\right)^{\lambda}=A_{1}^{\lambda} \otimes A_{2}^{\lambda} \lambda>0$

3. $\lambda_{1} A_{1} \oplus \lambda_{2} A_{1}=\left(\lambda_{1}+\lambda_{2}\right) A_{1}$ $A_{1}^{\lambda_{1}} \otimes A_{1}^{\lambda_{2}}=A_{1}^{\lambda_{1}+\lambda_{2}} \quad \lambda_{1}, \lambda_{2}>0$

Definition 3. [13] Let $A=\langle(a, b, c),(e, f, g),((r, s, t))\rangle$ be a SVTN number, the score function $S$ and accuracy function $H$ can be expressed as follows:

$$
\begin{gathered}
S(A)=\frac{1}{12}[8+(a+2 b+c)-(e+2 f+g)-(r+2 s+t)] \\
S(A) \in[0,1]
\end{gathered}
$$

$$
H(A)=\frac{1}{4}[(a+2 b+c)-(r+2 s+t)], H(A) \in[-1,1]
$$

Definition 4. [13] Let $A_{1}, A_{2}$ be two SVTN numbers, according Definition 3 the order relations are defined as follows:

1. if $S\left(A_{1}\right)<S\left(A_{2}\right)$, then $A_{1} \prec A_{2}$;

2. if $S\left(A_{1}\right)>S\left(A_{2}\right)$, then $A_{1}>A_{2}$;

3. if $S\left(A_{1}\right)=S\left(A_{2}\right), H\left(A_{1}\right)<H\left(A_{2}\right)$, then $A_{1} \prec A_{2}$;

4. if $S\left(A_{1}\right)=S\left(A_{2}\right), H\left(A_{1}\right)>H\left(A_{2}\right)$, then $A_{1}>A_{2}$;

5. if $S\left(A_{1}\right)=S\left(A_{2}\right), H\left(A_{1}\right)=H\left(A_{2}\right)$, then $A_{1} \sim A_{2}$;

\subsection{Dombi Operations}

Information aggregation in multi-criteria decision making is a crucial step. However, the existing aggregation operators are flexibility lack. To overcome this deficiency, Dombi [15] proposed Dombi operations, including T-norm and T-conorm.

Definition 5. [15] Let $s$ and $t$ be any two real numbers. Then, the Dombi T-norm and Dombi T-conorm among $s$ and $t$ are depicted as follows:

$$
\begin{gathered}
O_{D}(s, t)=\frac{1}{1+\left(\left(\frac{1-s}{s}\right)^{\gamma}+\left(\frac{1-t}{t}\right)^{\gamma}\right)^{1 / \gamma}} \\
O_{D}^{C}(s, t)=1-\frac{1}{1+\left(\left(\frac{s}{1-s}\right)^{\gamma}+\left(\frac{t}{1-t}\right)^{\gamma}\right)^{1 / \gamma}}
\end{gathered}
$$

where, $\gamma \geq 1$ and $(s, t) \in[0,1] \times[0,1]$.

\subsection{PA Operator}

When a decision maker makes a multi-criteria decision, all the criteria are not equally important, and there is a priority relationship between the criteria. Therefore, Yager [19] considering the priority relationship between the criteria proposed a PA operator.

Definition 6. [19] Let $C=\left\{C_{1}, C_{2}, \cdots, C_{n}\right\}$ be a set of criteria, and exists $C_{1}>C_{2}>\cdots>C_{n}$ priority relationship between the criteria. Where the value $y$ under the criteria $C_{i}$ is $C_{i}(y)(i=1,2, \cdots, n)$, and satisfies $C_{i}(y) \in[0,1]$. Then the PA operator is depicted as follows:

$$
P A\left(C_{1}(y), C_{2}(y), \cdots, C_{n}(y)\right)=\sum_{i=1}^{n} w_{i} C_{i}(y)
$$

where $w_{i}=\frac{H_{i}}{\sum_{i=1}^{n} H_{i}}, H_{i}=\prod_{k=1}^{i-1} C_{k}(y)(i \geq 2)$, and $H_{1}=1$.

\subsection{BM Operator}

In some special cases, the criteria are dependent criteria. In order to solve the relationship between the criteria, Bonferroni [24] proposed the BM aggregation operator.

Definition 7. [24] Let $b_{i}(i=1,2, \cdots, n)$ be a collection of nonnegative real numbers and $p, q>0$. The $\mathrm{BM}$ operator is depicted as follows:

$$
B M^{p, q}\left(b_{1}, b_{2}, \cdots, b_{n}\right)=\left(\frac{1}{n(n-1)} \sum_{\substack{i, j=1, i \neq j}}^{n} b_{i}^{p} b_{j}^{q}\right)^{1 / p+q}
$$

However, in practical problems, multi-criteria decision usually needs to consider the importance of the criteria, and assign different weights to different criteria. Yager et al. [25] defined WBM operator based on the BM operator. 
Definition 8. [25] Let $b_{i}(i=1,2, \cdots, n)$ be a collection of nonnegative real numbers and $p, q>0 . w=\left(w_{1}, w_{2}, \cdots w_{n}\right)^{T}$ is the weight vector of $b_{i}, \sum_{i=1}^{n} w_{i}=1$, and $w_{i} \in[0,1]$. The WBM operator is depicted as follows:

$W_{B M^{p}, q}\left(b_{1}, b_{2}, \cdots, b_{n}\right)=\left(\frac{1}{n(n-1)} \sum_{\substack{i, j=1, i \neq j}}^{n}\left(w_{i} b_{i}\right)^{p}\left(w_{j} b_{j}\right)^{q}\right)^{1 / p+q}$

There is a defect in this WBM operator that is non-reducibility. Zhou and He [26] further defined the NWBM operator based on the WBM operator.

Definition 9. [26] Let $b_{i}(i=1,2, \cdots, n)$ be a collection of nonnegative real numbers and $p, q>0 . w=\left(w_{1}, w_{2}, \cdots w_{n}\right)^{T}$ is the weight vector of $b_{i}, \sum_{i=1}^{n} w_{i}=1$, and $w_{i} \in[0,1]$. The NWBM operator is depicted as follows:

$\operatorname{NWBM}^{p, q}\left(b_{1}, b_{2}, \cdots, b_{n}\right)=\left(\sum_{\substack{i, j=1, i \neq j}}^{n} \frac{w_{i} w_{j}}{1-w_{i}} b_{i}^{p} b_{j}^{q}\right)^{1 / p+q}$

\section{DOMBI PRIORITIZED NORMALIZED BM OPERATOR OF SVTNS}

This section proposes SVTNDBM operator. Afterwards, we define the SVTNDPNBM operator on the basis of the Dombi operations, the PA operator and NWBM operator. The SVTNDPNBM operator has some flexibility, and simultaneously considers the priority relationship and interaction between the criteria by integrating the Dombi operations, the PA operator and NWBM operator. Then, we prove several properties of the SVTNDPNBM operator.

\subsection{Dombi Operations of SVTNS}

This part introduces Dombi operations of SVTNS based on the Definition 2 and the Definition 5.

Definition 10. Let

$A_{1}=\left\langle\left(a_{1}, b_{1}, c_{1}\right),\left(e_{1}, f_{1}, g_{1}\right),\left(r_{1}, s_{1}, t_{1}\right)\right\rangle, A_{2}=$ $\left\langle\left(a_{2}, b_{2}, c_{2}\right),\left(e_{2}, f_{2}, g_{2}\right),\left(r_{2}, s_{2}, t_{2}\right)\right\rangle$ be two SVTN numbers, $\gamma \geq 1$ and $\lambda>0$. Then, the Dombi T-norm and Dombi T-conorm of SVTNS can be depicted as follows:

1. $A_{1} \oplus_{D} A_{2}=\left\langle\left(1-\frac{1}{1+\left(\left(\frac{a_{1}}{1-a_{1}}\right)^{\gamma}+\left(\frac{a_{2}}{1-a_{2}}\right)^{\gamma}\right)^{\frac{1}{\gamma}}}, 1-\frac{1}{1+\left(\left(\frac{b_{1}}{1-b_{1}}\right)^{\gamma}+\left(\frac{b_{2}}{1-b_{2}}\right)^{\gamma}\right)^{\frac{1}{\gamma}}}, 1-\frac{1}{1+\left(\left(\frac{c_{1}}{1-c_{1}}\right)^{\gamma}+\left(\frac{c_{2}}{1-c_{2}}\right)^{\gamma}\right)^{\frac{1}{\gamma}}}\right)\right.$, $\left(\frac{1}{1+\left(\left(\frac{1-e_{1}}{e_{1}}\right)^{\gamma}+\left(\frac{1-e_{2}}{e_{2}}\right)^{\gamma}\right)^{\frac{1}{\gamma}}}, \frac{1}{1+\left(\left(\frac{1-f_{1}}{f_{1}}\right)^{\gamma}+\left(\frac{1-f_{2}}{f_{2}}\right)^{\gamma}\right)^{\frac{1}{\gamma}}}, \frac{1}{1+\left(\left(\frac{1-g_{1}}{g_{1}}\right)^{\gamma}+\left(\frac{1-g_{2}}{g_{2}}\right)^{\gamma}\right)^{\frac{1}{\gamma}}}\right)$,

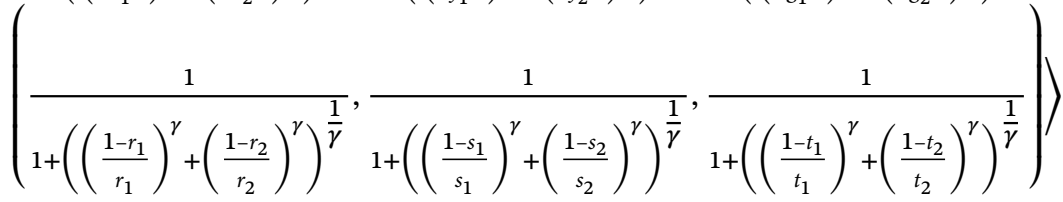

2. $A_{1} \otimes_{D} A_{2}=\left\langle\left(\frac{1}{1+\left(\left(\frac{1-a_{1}}{a_{1}}\right)^{\gamma}+\left(\frac{1-a_{2}}{a_{2}}\right)^{\gamma}\right)^{\frac{1}{\gamma}}}, \frac{1}{1+\left(\left(\frac{1-b_{1}}{b_{1}}\right)^{\gamma}+\left(\frac{1-b_{2}}{b_{2}}\right)^{\gamma}\right)^{\frac{1}{\gamma}}}, \frac{1}{1+\left(\left(\frac{1-c_{1}}{c_{1}}\right)^{\gamma}+\left(\frac{1-c_{2}}{c_{2}}\right)^{\gamma}\right)^{\frac{1}{\gamma}}}\right)\right.$,

$$
\begin{aligned}
& \left(1-\frac{1}{1+\left(\left(\frac{e_{1}}{1-e_{1}}\right)^{\gamma}+\left(\frac{e_{2}}{1-e_{2}}\right)^{\gamma}\right)^{\frac{1}{\gamma}}}, 1-\frac{1}{1+\left(\left(\frac{f_{1}}{1-f_{1}}\right)^{\gamma}+\left(\frac{f_{2}}{1-f_{2}}\right)^{\gamma}\right)^{\frac{1}{\gamma}}}, 1-\frac{1}{1+\left(\left(\frac{g_{1}}{1-g_{1}}\right)^{\gamma}+\left(\frac{g_{2}}{1-g_{2}}\right)^{\gamma}\right)^{\frac{1}{\gamma}}}\right) \text {, } \\
& \left.\left(1-\frac{1}{1+\left(\left(\frac{r_{1}}{1-r_{1}}\right)^{\gamma}+\left(\frac{r_{2}}{1-r_{2}}\right)^{\gamma}\right)^{\frac{1}{\gamma}}}, 1-\frac{1}{1+\left(\left(\frac{s_{1}}{1-s_{1}}\right)^{\gamma}+\left(\frac{s_{2}}{1-s_{2}}\right)^{\gamma}\right)^{\frac{1}{\gamma}}}, 1-\frac{1}{1+\left(\left(\frac{t_{1}}{1-t_{1}}\right)^{\gamma}+\left(\frac{t_{2}}{1-t_{2}}\right)^{\gamma}\right)^{\frac{1}{\gamma}}}\right)\right\rangle
\end{aligned}
$$


3. $\lambda \cdot{ }_{D} A_{1}=\left\langle\left(1-\frac{1}{1+\left(\lambda\left(\frac{a_{1}}{1-a_{1}}\right)^{\gamma}\right)^{\frac{1}{\gamma}}}, 1-\frac{1}{1+\left(\lambda\left(\frac{b_{1}}{1-b_{1}}\right)^{\gamma}\right)^{\frac{1}{\gamma}}}, 1-\frac{1}{1+\left(\lambda\left(\frac{c_{1}}{1-c_{1}}\right)^{\gamma}\right)^{\frac{1}{\gamma}}}\right)\right.$,

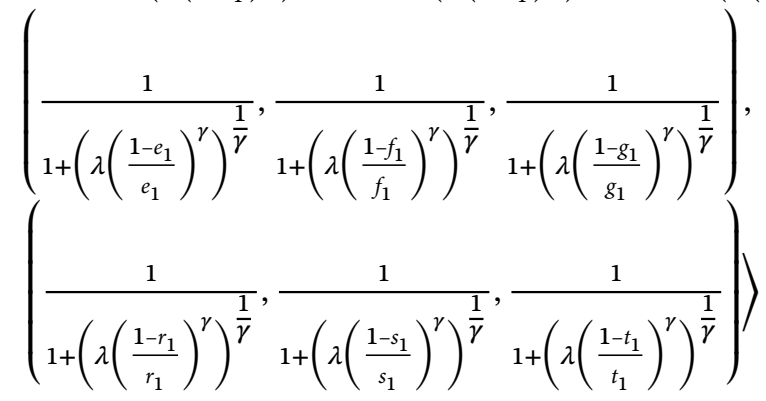

4.

$$
\begin{aligned}
& \left(A_{1}\right)^{\wedge_{D} \lambda}=\left\langle\left(\frac{1}{1+\left(\lambda\left(\frac{1-a_{1}}{a_{1}}\right)^{\gamma}\right)^{\frac{1}{\gamma}}}, \frac{1}{1+\left(\lambda\left(\frac{1-b_{1}}{b_{1}}\right)^{\gamma}\right)^{\frac{1}{\gamma}}}, \frac{1}{1+\left(\lambda\left(\frac{1-c_{1}}{c_{1}}\right)^{\gamma}\right)^{\frac{1}{\gamma}}}\right),\right.
\end{aligned}
$$

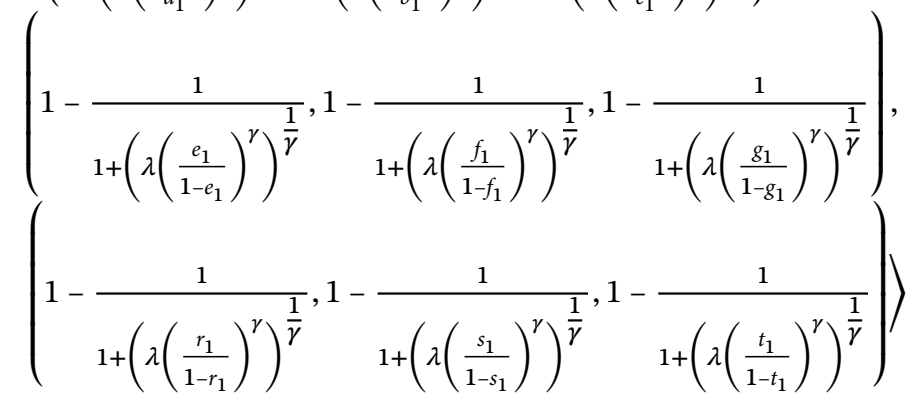

\subsection{The SVTNDPNBM Operator}

Now, based on these new Dombi T-norm and Dombi T-conorm of SVTNS, we define the SVTNDBM operator and the SVTNDPNBM operator.

\section{Definition 11. Let}

$x_{i}=\left\langle\left(a_{i}, b_{i}, c_{i}\right),\left(e_{i}, f_{i}, g_{i}\right),\left(r_{i}, s_{i}, t_{i}\right)\right\rangle(i=1,2, \cdots, n)$ be a set of SVTN numbers, and $p, q>0$. Then, the SVTNDBM operator can be depicted as follows:

$$
\begin{aligned}
& \operatorname{SVTNDBM}^{p, q}\left(x_{1}, x_{2}, \cdots, x_{n}\right)= \\
& \left(\frac{1}{n(n-1)} \cdot{ }_{D} \bigoplus_{\substack{i, j=1, i \neq j}}^{n}\left(\left(x_{i}\right)^{\wedge_{D} p} \otimes_{D}\left(x_{j}\right)^{\wedge_{D} q}\right)\right)^{\wedge_{D} \frac{1}{p+q}}
\end{aligned}
$$

This part proposes the SVTNDPNBM operator based on the PA operator and NWBM operator as Definitions 6 and 9. The SVTNDPNBM operator is defined as follows:
Definition 12. Let $C=\left\{C_{1}, C_{2}, \cdots, C_{n}\right\}$ be a set of criteria, and exists $C_{1}>C_{2}>\cdots>C_{n}$ priority relationship between the criteria. The performance value of object $x$ under criterion $C_{i}$ is denoted by SVTN numbers $x_{i}=$ $\left\langle\left(a_{i}, b_{i}, c_{i}\right),\left(e_{i}, f_{i}, g_{i}\right),\left(r_{i}, s_{i}, t_{i}\right)\right\rangle(i=1,2, \cdots, n)$. Then the SVTNDPNBM operator is depicted as follows:

$$
\begin{aligned}
& \operatorname{SVTNDPNBM} M^{p, q}\left(x_{1}, x_{2}, \cdots, x_{n}\right)= \\
& \left({\substack{i, j=1, \oplus_{D} \\
i \neq j}}_{n}^{n}\left(\frac{w_{i} w_{j}}{1-w_{i}} \cdot{ }_{D}\left(\left(x_{i}\right)^{\wedge_{D} p} \otimes_{D}\left(x_{j}\right)^{\wedge_{D} q}\right)\right)\right)^{\wedge_{D} \frac{1}{p+q}}
\end{aligned}
$$

where $w_{i}=\frac{H_{i}}{\sum_{i=1}^{n} H_{i}}, H_{i}=\coprod_{k=1}^{i-1} S\left(x_{k}\right)(i \geq 2), H_{1}=1$, and $S\left(x_{k}\right)$ is the score function of SVTN number $x_{k}$ obtained by Definition 4 .

Theorem 1. The SVTNDPNBM operator in Definition 12 is still an SVTN number, an 


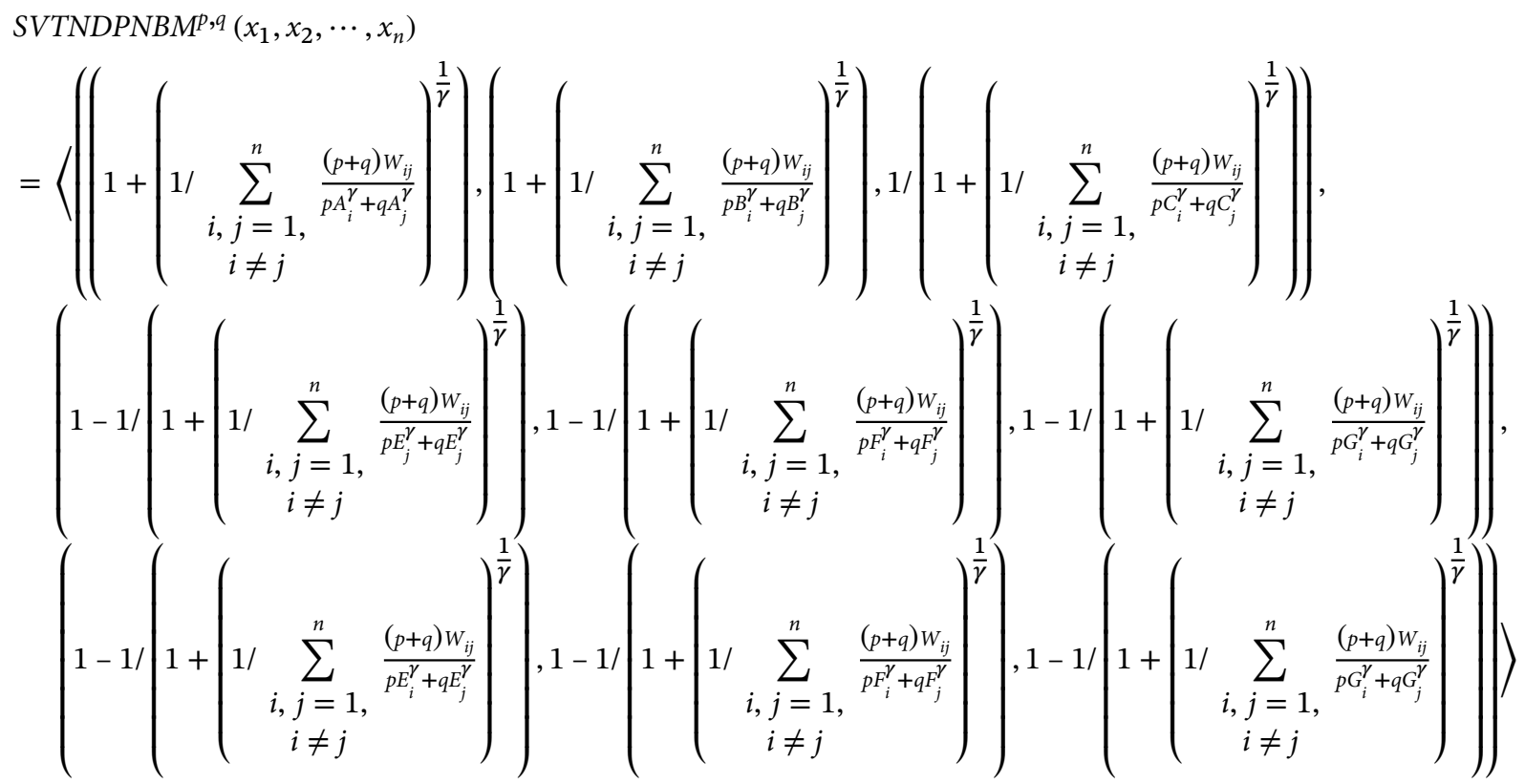

Theorem 1 can be proved by mathematical operations as follows:

Proof. Let $\frac{1-a_{i}}{a_{i}}=A_{i}, \frac{1-a_{j}}{a_{j}}=A_{j}, \frac{1-b_{i}}{b_{i}}=B_{i}, \frac{1-b_{j}}{b_{j}}=B_{j}$, $\frac{1-c_{i}}{c_{i}}=C_{i}, \frac{1-c_{j}}{c_{j}}=C_{j}, \frac{e_{i}}{1-e_{i}}=E_{i}, \frac{e_{j}}{1-e_{j}}=E_{j}, \frac{f_{i}}{1-f_{i}}=F_{i}$, $\frac{f_{j}}{1-f_{j}}=F_{j}, \frac{g_{i}}{1-g_{i}}=G_{i}, \frac{g_{j}}{1-g_{j}}=G_{j}, \frac{r_{i}}{1-r_{i}}=R_{i}, \frac{r_{j}}{1-r_{j}}=R_{j}$, $\frac{s_{i}}{1-s_{i}}=S_{i}, \frac{s_{j}}{1-s_{j}}=S_{j}, \frac{t_{i}}{1-t_{i}}=T_{i}, \frac{t_{j}}{1-t_{j}}=T_{j}, \frac{w_{i} w_{j}}{1-w_{i}}=W_{i j}$. According to Definition 10, we have,

$\left(x_{i}\right)^{\wedge_{D} P} \otimes_{D}\left(x_{j}\right)^{\wedge_{D} q}=$

$\left\langle\left(\frac{1}{1+\left(p A_{i}^{\gamma}+q A_{j}^{\gamma}\right)^{\frac{1}{\gamma}}}, \frac{1}{1+\left(p B_{i}^{\gamma}+q B_{j}^{\gamma}\right)^{\frac{1}{\gamma}}}, \frac{1}{1+\left(p C_{i}^{\gamma}+q C_{j}^{\gamma}\right)^{\frac{1}{\gamma}}}\right)\right.$,

$\left(1-\frac{1}{1+\left(p E_{i}^{\gamma}+q E_{j}^{\gamma}\right)^{\frac{1}{\gamma}}}, 1-\frac{1}{1+\left(p F_{i}^{\gamma}+q F_{j}^{\gamma}\right)^{\frac{1}{\gamma}}}\right.$,

$\left.1-\frac{1}{1+\left(p G_{i}^{\gamma}+q G_{j}^{\gamma}\right)^{\frac{1}{\gamma}}}\right),\left(1-\frac{1}{1+\left(p R_{i}^{\gamma}+q R_{j}^{\gamma}\right)^{\frac{1}{\gamma}}}\right.$,

$\left.\left.1-\frac{1}{1+\left(p S_{i}^{\gamma}+q S_{j}^{\gamma}\right)^{\frac{1}{\gamma}}}, 1-\frac{1}{1+\left(p T_{i}^{\gamma}+q T_{j}^{\gamma}\right)^{\frac{1}{\gamma}}}\right)\right\rangle$
Then,

$\frac{w_{i} w_{j}}{1-w_{i}} \cdot{ }_{D}\left(x_{i}\right)^{\wedge_{D} p} \otimes_{D}\left(x_{j}\right)^{\wedge_{D} p}=\left\langle\left(1-1 /\left(1+\frac{W_{i j}^{\frac{1}{\gamma}}}{\left(p A_{i}^{\gamma}+q A_{j}^{\gamma}\right)^{\frac{1}{\gamma}}}\right)\right.\right.$,

$\left.1-1 /\left(1+\frac{W_{i j}^{\frac{1}{\gamma}}}{\left(p B_{i}^{\gamma}+q B_{j}^{\gamma}\right)^{\frac{1}{\gamma}}}\right), 1-1 /\left(1+\frac{W_{i j}^{\frac{1}{\gamma}}}{\left(p C_{i}^{\gamma}+q C_{j}^{\gamma}\right)^{\frac{1}{\gamma}}}\right)\right)$,

$\left(1 /\left(1+\frac{W_{i j}^{\frac{1}{\gamma}}}{\left(p E_{i}^{\gamma}+q E_{j}^{\gamma}\right)^{\frac{1}{\gamma}}}\right), 1 /\left(1+\frac{W_{i j}^{\frac{1}{\gamma}}}{\left(p F_{i}^{\gamma}+q F_{j}^{\gamma}\right)^{\frac{1}{\gamma}}}\right)\right.$,

$\left.1 /\left(1+\frac{W_{i j}^{\frac{1}{\gamma}}}{\left(p G_{i}^{\gamma}+p G_{j}^{\gamma}\right)^{\frac{1}{\gamma}}}\right)\right),\left(1 /\left(1+\frac{W_{i j}^{\frac{1}{\gamma}}}{\left(p R_{i}^{\gamma}+q R_{j}^{\gamma}\right)^{\frac{1}{\gamma}}}\right)\right.$,

$\left.1 /\left(1+\frac{W_{i j}^{\frac{1}{\gamma}}}{\left(p S_{i}^{\gamma}+q S_{j}^{\gamma}\right)^{\frac{1}{\gamma}}}\right), 1 /\left(1+\frac{W_{i j}^{\frac{1}{\gamma}}}{\left(p T_{i}^{\gamma}+q T_{j}^{\gamma}\right)^{\frac{1}{\gamma}}}\right)\right)$ 
And,

$$
\begin{aligned}
& \bigoplus_{i, j=1,}^{n}\left(\frac{w_{i} w_{j}}{1-w_{i}} \cdot{ }_{D}\left(\left(x_{i}\right)^{\wedge_{D} P} \otimes_{D}\left(x_{j}\right)^{\wedge_{D} q}\right)\right) \\
& i \neq j \\
& =\left\langle 1-1 /\left(1+\left(\sum_{\substack{i, j=1, i \neq j}}^{n} \frac{W_{i j}}{p A_{i}^{\gamma}+q A_{j}^{\gamma}}\right)^{\frac{1}{\gamma}}\right), 1-1 /\left(1+\left(\sum_{\substack{i, j=1, i \neq j}}^{n} \frac{W_{i j}}{p B_{i}^{\gamma}+q B_{j}^{\gamma}}\right)^{\frac{1}{\gamma}}\right), 1-1 /\left(1+\left(\sum_{\substack{i, j=1, i \neq j}}^{n} \frac{W_{i j}}{p C_{i}^{\gamma}+q C_{j}^{\gamma}}\right)\right)\right. \\
& 1 /\left(1+\left(\sum_{\substack{i, j=1, i \neq j}}^{n} \frac{W_{i j}}{p E_{i}^{\gamma}+p E_{j}^{\gamma}}\right)^{\frac{1}{\gamma}}, 1 /\left(1+\left(\sum_{\substack{i, j=1, i \neq j}}^{n} \frac{W_{i j}}{p F_{i}^{\gamma}+q F_{j}^{\gamma}}\right)^{\frac{1}{\gamma}}\right), 1 /\left(1+\left(\sum_{\substack{i, j=1, i \neq j}}^{n} \frac{W_{i j}}{p G_{i}^{\gamma}+q G_{j}^{\gamma}}\right)^{\frac{1}{\gamma}}\right),\right. \\
& 1 /\left(1+\left(\sum_{\substack{i, j=1, i \neq j}}^{n} \frac{W_{i j}}{p R_{i}^{\gamma}+q R_{j}^{\gamma}}\right)^{\frac{1}{\gamma}}, 1 /\left(1+\left(\sum_{\substack{i, j=1, i \neq j}}^{n} \frac{W_{i j}}{p S_{i}^{\gamma}+q S_{j}^{\gamma}}\right)^{\frac{1}{\gamma}}\right), 1 /\left(1+\left(\sum_{\substack{i, j, 1 \\
i \neq j}}^{n} \frac{W_{i j}}{p T_{i}^{\gamma}+q T_{j}^{\gamma}}\right)\right)\right.
\end{aligned}
$$

Furthermore, according to Definition 10 we can prove that Theorem 1 is established.

\section{Theorem 2. (Reducibility)}

Let $C=\left\{C_{1}, C_{2}, \cdots, C_{n}\right\}$ be a set of criteria, and $C_{i}(i=1,2, \cdots, n)$ have the priority relationship. When $w_{i}=\frac{1}{n}(i=1,2, \cdots, n)$ is satisfied, the SVTNDPNBM operator is equivalent to the SVTNDBM operator.

Proof. When $w_{i}=\frac{1}{n}(i=1,2, \cdots, n)$, then $\frac{w_{i} w_{j}}{1-w_{i}}=\frac{1}{n(n-1)}$, according to Definition 10 , we can obtain that

$$
\operatorname{SVTNDPNBM}^{p, q}\left(x_{1}, x_{2}, \cdots, x_{n}\right)=\operatorname{SVTNDBM}^{p, q}\left(x_{1}, x_{2}, \cdots, x_{n}\right)
$$

Then, we can prove that Theorem 2 is established.

Theorem 3. (Idempotency)

Let $x_{i}=\left\langle\left(a_{i}, b_{i}, c_{i}\right),\left(e_{i}, f_{i}, g_{i}\right),\left(r_{i}, s_{i}, t_{i}\right)\right\rangle(i=1,2, \cdots, n)$ be a set of $S V T N$ numbers, if all SVTN number are equal, i.e., $x_{i}=x$. Then,

$$
\operatorname{SVTNDPNBM}^{p, q}\left(x_{1}, x_{2}, \cdots, x_{n}\right)=x
$$

Proof. When $x_{i}=x$, according to Definition 10, we can obtain that

$$
\begin{aligned}
& \operatorname{SVTNDPNBM}^{p, q}\left(x_{1}, x_{2}, \cdots, x_{n}\right) \\
& =\left(\bigoplus_{\substack{i, j=1, i \neq j}}^{n}\left(\frac{w_{i} w_{j}}{1-w_{i}} \cdot{ }_{D}\left(\left(x_{i}\right)^{\wedge_{D} p} \otimes_{D}\left(x_{j}\right)^{\wedge_{D} q}\right)\right)\right)^{\wedge_{D} \frac{1}{p+q}}
\end{aligned}
$$

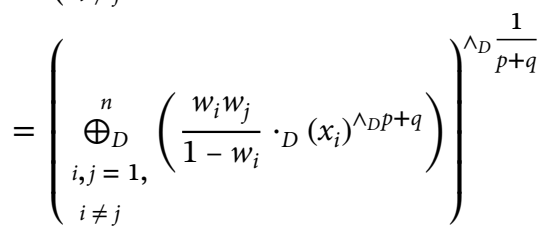

$$
\begin{aligned}
& =\left(\sum_{\substack{i, j=1, i \neq j}}^{n} \frac{w_{i} w_{j}}{1-w_{i}} \cdot{ }_{D}\left(x_{i}\right)^{\wedge_{D} p+q}\right)^{\wedge_{D} \frac{1}{p+q}} \\
& =\left(\left(x_{i}\right)^{\wedge_{D} p+q}\right)^{\wedge_{D} \frac{1}{p+q}} \\
& =x
\end{aligned}
$$

Then, we can prove that Theorem 3 is established. 


\section{METHOD FOR SELECTING GREEN SUPPLIER}

Suppose that there are $m$ green providers $X=\left\{x_{1}, x_{2}, \cdots, x_{m}\right\}$ and $n$ criteria $C=\left\{c_{1}, c_{2}, \cdots, c_{n}\right\}$. There is a correlation between the criteria, and different criteria have a strict priority relationship. Let $U=\left(a_{i j}\right)_{m \times n}$ be a single-valued triangular Neutrosophic decision matrix, where $a_{i j}=\left\langle T_{i j}, I_{i j}, F_{i j}\right\rangle$ is the evaluation value of each green supplier under different criteria.

The following section is a sorting process for green suppliers based on the SVTNDPNBM operator.

- Step 1: Calculate the score value $s_{i j}$ of each $a_{i j}$ according to Definition 3.

- Step 2: Determine the relevant weight according to each $s_{i j}$.

- Step 3: Assume $\gamma=1, p=q=1$ and aggregate the evaluation values of each green suppliers based on Definition 12 and the results of Step 2.

- Step 4: Calculate the value $S\left(x_{i}\right), H\left(x_{i}\right)(i=1,2, \cdots, m)$ of the green suppliers after aggregation according to Definition 3.

- Step 5: Sort by the results of step 4 .

- Step 6: Replace $\gamma, p, q$ with different values and compare analysis.

- Step 7: Comparative analysis.

\section{NUMERICAL EXAMPLE}

Consider the green supplier selection problem in which a criterion has a priority relationship and a mutual relationship and cannot give corresponding weights to the example of the section. There are five green suppliers to choose $X=\left\{x_{1}, x_{2}, \cdots, x_{5}\right\}$ and five evaluation criteria $C=\left\{c_{1}, c_{2}, \cdots, c_{5}\right\}$. The five criteria are product quality, cost, service capability, green image, and innovation capability. The priority relationship between the criteria is $c_{1}>c_{2}>c_{3}>c_{4}>c_{5}$. Table 1 is a single-valued triangular Neutrosophic decision matrix. The elements in the Table 1 represent the corresponding evaluation values under the five criteria.

Step 1: Calculate the score value $s_{i j}$ of each $a_{i j}$ according to Definition 3. The calculation results are shown in Table 2.
Step 2: Determine the relevant weight according to each $s_{i j}$. The calculation results are shown in Table 3.

Step 3: Assume $\gamma=1, p=q=1$ and aggregate the evaluation values of each green suppliers based on Definition 12 and the results of Step 2. According to this, the comprehensive evaluation value of the green suppliers can be obtained as follows:

$$
\begin{aligned}
& x_{1}=\left\langle\begin{array}{l}
(0.5319,0.6211,0.7872),(0.3547,0.4150,0.5237), \\
(0.1884,0.3404,0.4666)
\end{array}\right\rangle \\
& x_{2}=\left\langle\begin{array}{l}
(0.5720,0.6539,0.7654),(0.3966,0.4953,0.6066), \\
(0.2050,0.3372,0.4159)
\end{array}\right\rangle \\
& x_{3}=\left\langle\begin{array}{l}
(0.6490,0.7340,0.8307),(0.4666,0.5469,0.6115), \\
(0.3559,0.4516,0.5349)
\end{array}\right\rangle \\
& x_{4}=\left\langle\begin{array}{l}
(0.7449,0.8064,0.9390),(0.5704,0.6347,0.7543), \\
(0.4050,0.5156,0.6033)
\end{array}\right\rangle \\
& x_{5}=\left\langle\begin{array}{l}
(0.5351,0.6188,0.7876),(0.4351,0.4919,0.5705), \\
(0.2919,0.3808,0.4983)
\end{array}\right\rangle
\end{aligned}
$$

Step 4: Calculate the value $S\left(x_{i}\right), H\left(x_{i}\right)(i=1,2, \cdots, m)$ of the green suppliers after aggregation according to Definition 3. And the calculation results are shown in Table 4.

Step 5: Sort by the results of step 4.

Since $S\left(x_{1}\right)>S\left(x_{2}\right)>S\left(x_{5}\right)>S\left(x_{3}\right)>S\left(x_{4}\right)$, according to Definition 4 , we sort the green suppliers as $x_{1}>x_{2}>x_{5}>x_{3}>x_{4}$.

Step 6: Replace $\gamma, p, q$ with different values and compare analysis.

In order to consider the influence of parameters on the ordering, this step analyzes the influence of the change of $\gamma$ on the ordering when $p, q=1$, and the effect of the change of $p, q$ on the ordering when $\gamma=1$. The effect of the change of $\gamma$ is shown in Table 5, and the results of the change of $p, q$ are shown in Table 6.

\section{Step 7: Comparative analysis}

Compare the method proposed in this paper with the methods in other literatures, the results are shown in Table 7.

Table 1 Single-valued triangular Neutrosophic decision matrix.

\begin{tabular}{cllllll}
\hline & \multicolumn{1}{c}{$\boldsymbol{c}_{\mathbf{1}}$} & \multicolumn{1}{c}{$\boldsymbol{c}_{\mathbf{2}}$} & \multicolumn{1}{c}{$\boldsymbol{c}_{\mathbf{3}}$} & \multicolumn{1}{c}{$\boldsymbol{c}_{\mathbf{4}}$} & \multicolumn{1}{c}{$\boldsymbol{c}_{\mathbf{5}}$} \\
\hline$x_{\mathbf{1}}$ & $<(0.42,0.53,0.71)$, & $<(0.80,0.86,0.92)$, & $<(0.42,0.52,0.81)$, & $<(0.51,0.55,0.58)$, & $<(0.72,0.74,0.85)$, \\
& $(0.38,0.45,0.53)$, & $(0.26,0.28,0.37)$, & $(0.41,0.45,0.58)$, & $(0.25,0.37,0.57)$, & $(0.62,0.68,0.72)$, \\
& $(0.21,0.34,0.46)>$ & $(0.05,0.18,0.24)>$ & $(0.34,0.42,0.61)>$ & $(0.15,0.47,0.62)>$ & $(0.42,0.48,0.52)>$ \\
$x_{2}$ & $<(0.50,0.55,0.64)$, & $<(0.71,0.84,0.86)$, & $<(0.40,0.51,0.67)$, & $<(0.80,0.82,0.86)$, & $<(0.57,0.61,0.97)$, \\
& $(0.25,0.37,0.42)$, & $(0.51,0.58,0.64)$, & $(0.40,0.51,0.67)$, & $(0.42,0.51,0.67)$, & $(0.53,0.61,0.84)$, \\
& $(0.20,0.29,0.31)>$ & $(0.12,0.35,0.42)>$ & $(0.34,0.45,0.61)>$ & $(0.21,0.25,0.38)>$ & $(0.28,0.36,0.42)>$ \\
$x_{3}$ & $<(0.75,0.84,0.91)$, & $<(0.64,0.69,0.85)$, & $<(0.54,0.68,0.72)$, & $<(0.50,0.58,0.61)$, & $<(0.71,0.75,0.82)$, \\
& $(0.54,0.62,0.67)$, & $(0.34,0.45,0.52)$, & $(0.45,0.48,0.51)$, & $(0.42,0.51,0.67)$, & $(0.61,0.68,0.72)$, \\
& $(0.47,0.57,0.61)>$ & $(0.21,0.29,0.38)>$ & $(0.35,0.39,0.42)>$ & $(0.31,0.38,0.56)>$ & $(0.31,0.58,0.86)>$ \\
$x_{4}$ & $<(0.85,0.89,0.94)$, & $<(0.57,0.61,0.97)$, & $<(0.87,0.89,0.94)$, & $<(0.64,0.84,0.87)$, & $<(0.47,0.51,0.67)$, \\
& $(0.65,0.67,0.75)$, & $(0.53,0.61,0.84)$, & $(0.54,0.68,0.71)$, & $(0.51,0.58,0.64)$, & $(0.32,0.45,0.58)$, \\
& $(0.61,0.69,0.76)>$ & $(0.28,0.36,0.42)>$ & $(0.23,0.35,0.51)>$ & $(0.32,0.38,0.42)>$ & $(0.12,0.38,0.45)>$ \\
$x_{5}$ & $<(0.42,0.52,0.81)$, & $<(0.51,0.58,0.64)$, & $<(0.72,0.74,0.85)$, & $<(0.71,0.84,0.86)$, & $<(0.64,0.69,0.85)$, \\
& $(0.41,0.45,0.58)$, & $(0.34,0.39,0.41)$, & $(0.62,0.68,0.72)$, & $(0.51,0.58,0.64)$, & $(0.34,0.45,0.52)$, \\
& $(0.34,0.42,0.61)>$ & $(0.24,0.29,0.34)>$ & $(0.42,0.48,0.52)>$ & $(0.12,0.35,0.42)>$ & $(0.21,0.29,0.38)>$ \\
\hline
\end{tabular}


Table 2 Score value.

\begin{tabular}{cccccc}
\hline & $\boldsymbol{c}_{\mathbf{1}}$ & $\boldsymbol{c}_{\mathbf{2}}$ & $\boldsymbol{c}_{\mathbf{3}}$ & $\boldsymbol{c}_{\boldsymbol{4}}$ & $\boldsymbol{c}_{\mathbf{5}}$ \\
\hline$x_{\mathbf{1}}$ & 0.5858 & 0.800 & 0.5492 & 0.5767 & 0.5375 \\
$x_{2}$ & 0.6450 & 0.6417 & 0.5125 & 0.6750 & 0.5625 \\
$x_{\mathbf{3}}$ & 0.5558 & 0.6617 & 0.5958 & 0.5442 & 0.5008 \\
$x_{\mathbf{4}}$ & 0.5067 & 0.5625 & 0.6283 & 0.6150 & 0.5858 \\
$x_{5}$ & 0.5492 & 0.6350 & 0.5375 & 0.6417 & 0.6617 \\
\hline
\end{tabular}

Table 3 Weight matrix.

\begin{tabular}{cccccc}
\hline & $c_{\mathbf{1}}$ & $\boldsymbol{c}_{\mathbf{2}}$ & $\boldsymbol{c}_{\mathbf{3}}$ & $\boldsymbol{c}_{\mathbf{4}}$ & $\boldsymbol{c}_{\mathbf{5}}$ \\
\hline$x_{1}$ & 0.4065 & 0.2381 & 0.1905 & 0.1046 & 0.0603 \\
$x_{2}$ & 0.4142 & 0.2672 & 0.1714 & 0.0879 & 0.0593 \\
$x_{3}$ & 0.4421 & 0.2457 & 0.1626 & 0.0969 & 0.0527 \\
$x_{4}$ & 0.4806 & 0.2435 & 0.1369 & 0.0861 & 0.0529 \\
$x_{5}$ & 0.4534 & 0.2490 & 0.1581 & 0.0850 & 0.0545 \\
\hline
\end{tabular}

Table 4 Score value and accuracy value.

\begin{tabular}{cccccc}
\hline & $\boldsymbol{x}_{\mathbf{1}}$ & $\boldsymbol{x}_{\mathbf{2}}$ & $\boldsymbol{x}_{\mathbf{3}}$ & $\boldsymbol{x}_{\mathbf{4}}$ & $\boldsymbol{x}_{\mathbf{5}}$ \\
\hline$S\left(x_{i}\right)$ & 0.6264 & 0.6130 & 0.5818 & 0.5553 & 0.5849 \\
$H\left(x_{i}\right)$ & 0.3064 & 0.3375 & 0.2885 & 0.3143 & 0.2521 \\
\hline
\end{tabular}

Table 5 The ranking orders of different $\gamma$.

\begin{tabular}{ccc}
\hline$\gamma$ & Score Value & Ranking Order \\
\hline 1 & $S\left(x_{1}\right)=0.6264, S\left(x_{2}\right)=0.6130, S\left(x_{3}\right)=0.5818, S\left(x_{4}\right)=0.5553, S\left(x_{5}\right)=0.5849$ & $x_{1}>x_{2}>x_{5}>x_{3}>x_{4}$ \\
2 & $S\left(x_{1}\right)=0.6194, S\left(x_{2}\right)=0.6097, S\left(x_{3}\right)=0.5776, S\left(x_{4}\right)=0.5628, S\left(x_{5}\right)=0.5835$ & $x_{1}>x_{2}>x_{5}>x_{3}>x_{4}$ \\
5 & $S\left(x_{1}\right)=0.6302, S\left(x_{2}\right)=0.6275, S\left(x_{3}\right)=0.5849, S\left(x_{4}\right)=0.5956, S\left(x_{5}\right)=0.6001$ & $x_{1}>x_{2}>x_{5}>x_{4}>x_{3}$ \\
10 & $S\left(x_{1}\right)=0.6518, S\left(x_{2}\right)=0.6436, S\left(x_{3}\right)=0.6043, S\left(x_{4}\right)=0.6165, S\left(x_{5}\right)=0.6272$ & $x_{1}>x_{2}>x_{5}>x_{4}>x_{3}$ \\
50 & $S\left(x_{1}\right)=0.6789, S\left(x_{2}\right)=0.6610, S\left(x_{3}\right)=0.6314, S\left(x_{4}\right)=0.6469, S\left(x_{5}\right)=0.6654$ & $x_{1}>x_{2}>x_{5}>x_{4}>x_{3}$ \\
\hline
\end{tabular}

Table 6 The ranking orders of different $p, q$.

\begin{tabular}{ccc}
\hline $\boldsymbol{p}, \boldsymbol{q}$ & Score Value & Ranking Order \\
\hline $0.001,1$ & $S\left(x_{1}\right)=0.6825, S\left(x_{2}\right)=0.6518, S\left(x_{3}\right)=0.6139, S\left(x_{4}\right)=0.6055, S\left(x_{5}\right)=0.6198$ & $x_{1}>x_{2}>x_{5}>x_{3}>x_{4}$ \\
$0.01,1$ & $S\left(x_{1}\right)=0.6800, S\left(x_{2}\right)=0.6499, S\left(x_{3}\right)=0.6126, S\left(x_{4}\right)=0.6032, S\left(x_{5}\right)=0.6185$ & $x_{1}>x_{2}>x_{5}>x_{3}>x_{4}$ \\
$0.1,1$ & $S\left(x_{1}\right)=0.6614, S\left(x_{2}\right)=0.6363, S\left(x_{3}\right)=0.6025, S\left(x_{4}\right)=0.5868, S\left(x_{5}\right)=0.6080$ & $x_{1}>x_{2}>x_{5}>x_{3}>x_{4}$ \\
1,1 & $S\left(x_{1}\right)=0.6264, S\left(x_{2}\right)=0.6130, S\left(x_{3}\right)=0.5818, S\left(x_{4}\right)=0.5553, S\left(x_{5}\right)=0.5849$ & $x_{1}>x_{2}>x_{5}>x_{3}>x_{4}$ \\
1,2 & $S\left(x_{1}\right)=0.6322, S\left(x_{2}\right)=0.6159, S\left(x_{3}\right)=0.5854, S\left(x_{4}\right)=0.5613, S\left(x_{5}\right)=0.5896$ & $x_{1}>x_{2}>x_{5}>x_{3}>x_{4}$ \\
1,5 & $S\left(x_{1}\right)=0.6489, S\left(x_{2}\right)=0.6274, S\left(x_{3}\right)=0.5954, S\left(x_{4}\right)=0.5759, S\left(x_{5}\right)=0.6004$ & $x_{1}>x_{2}>x_{5}>x_{3}>x_{4}$ \\
1,10 & $S\left(x_{1}\right)=0.6614, S\left(x_{2}\right)=0.6363, S\left(x_{3}\right)=0.6025, S\left(x_{4}\right)=0.5868, S\left(x_{5}\right)=0.6080$ & $x_{1}>x_{2}>x_{5}>x_{3}>x_{4}$ \\
$0.1,0.1$ & $S\left(x_{1}\right)=0.6264, S\left(x_{2}\right)=0.6130, S\left(x_{3}\right)=0.5818, S\left(x_{4}\right)=0.5553, S\left(x_{5}\right)=0.5849$ & $x_{1}>x_{2}>x_{5}>x_{3}>x_{4}$ \\
4,4 & $S\left(x_{1}\right)=0.6264, S\left(x_{2}\right)=0.6130, S\left(x_{3}\right)=0.5818, S\left(x_{4}\right)=0.5553, S\left(x_{5}\right)=0.5849$ & $x_{1}>x_{2}>x_{5}>x_{3}>x_{4}$ \\
10,10 & $S\left(x_{1}\right)=0.6264, S\left(x_{2}\right)=0.6130, S\left(x_{3}\right)=0.5818, S\left(x_{4}\right)=0.5553, S\left(x_{5}\right)=0.5849$ & $x_{1}>x_{2}>x_{5}>x_{3}>x_{4}$ \\
\hline
\end{tabular}

\begin{tabular}{l|l} 
Table 7 & The ranking orders of approaches.
\end{tabular}

\begin{tabular}{lc}
\hline & Order \\
\hline SVTNDPNBM & $x_{1}>x_{2}>x_{5}>x_{3}>x_{4}$ \\
[13] TFNNWA & $x_{1}>x_{2}>x_{3}>x_{5}>x_{4}$ \\
[13] TFNNWG & $x_{1}>x_{2}>x_{5}>x_{3}>x_{4}$ \\
[14] VIKOR & $x_{1}>x_{2}>x_{3}>x_{5}>x_{4}$ \\
\hline
\end{tabular}

As shown in Tables 5 and 6, the best green supplier is always $x_{1}$, no matter how the parameters change. As shown in Table 7, the best green supplier is always $x_{1}$, no matter which method is used. These results demonstrate that the applicability and stability of the SVTNDPNBM operator proposed in this paper.

\section{CONCLUSION}

In this paper, we use SVTNS to indicate the evaluation value, and use the triangular fuzzy number to represent the truth-membership function, the indeterminacy-membership function and the falsity-membership function, which can retain more uncertain information of the object to be evaluated. The main contribution of this paper is to consider the advantages and flexibility of Dombi operations, PA operator, and BM operator, and combine them to propose the SVTNDPNBM operator. The new aggregate operator takes into account the priority relationship and the interrelationship between the criteria. To make the new aggregate operator more flexible, this paper introduces the Dombi operations. The 
feasibility of the SVTNDPNBM operator is verified by the example chosen by the green supplier, and the stability of the SVTNDPNBM operator is verified by the change of the parameters.

In the future, due to the variety of operators, we can combine different operators and propose new operators. At the same time, we can further explore the different properties and applications of the SVTNS.

\section{CONFLICT OF INTEREST}

We declare that we do not have conflicts of interest with the work submitted.

\section{AUTHORS' CONTRIBUTIONS}

Funding acquisition was done by Mei Qin Wu; methodology prepared by Jian Ping Fan; original draft written, edited, and reviewed by Xuefei Jia.

\section{Funding Statement}

This work was supported by the Fund for Shanxi "1331 Project" Key Innovative Research Team (2017) and Shanxi "Disciplinary Group Construction Plan for Industry Innovation": Management of Intelligent Logistics System (2018).

\section{REFERENCES}

[1] L.A. Zadeh, Fuzzy sets, Inf. Control 8 (1965), 338-353.

[2] K.T. Atanassov, Intuitionistic fuzzy sets, Fuzzy Set. Syst. 20 (1986), $87-96$.

[3] K.T. Atanassov, G. Gargov, Interval valued intuitionistic fuzzy sets, Fuzzy Sets, 31 (1989), 343-349.

[4] K.T. Atanassov, Operators over interval valued intuitionistic fuzzy sets, Fuzzy Set. Syst. 64 (1994), 159-174.

[5] V. Torra, Hesitant fuzzy sets, Int. J. Intell. Syst. 25 (2010), 529-539.

[6] G. Qian, H. Wang, X. Feng, Generalized hesitant fuzzy sets and their application in decision support system, Knowl. Based Syst. 37 (2013), 357-365.

[7] B. Zhu, Z. Xu, M. Xia, Dual hesitant fuzzy sets, J. Appl. Math. 2012 (2012), 2607-2645.

[8] R.R. Yager, Pythagorean membership grades in multicriteria decision making, IEEE Trans. Fuzzy Syst. 22 (2014), 958-965.

[9] F. Smarandache, A Unifying Field in Logics. Neutrosophy: neutrosophic Probability, Set and Logic, American Research Press, Rehoboth, 1999. http://www.gallup.unm.edu/ smarandache/ eBooks-otherformats.htm

[10] J. Ye, Vector similarity measures of simplified neutrosophic sets and their application in multicriteria decision making, Int. J. Fuzzy Syst. 16 (2014), 204-211. http://vixra.org/pdf/1411. 0521v1.pdf

[11] J. Ye, A multicriteria decision-making method using aggregation operators for simplified Neutrosophic sets, J. Intell. Fuzzy Syst. 26 (2014), 2459-2466.

[12] J.J. Peng, J.Q. Wang, J. Wang, et al., Simplified neutrosophic sets and their applications in multi-criteria group decision-making problems. Int. J. Syst. Sci. 47 (2016), 2342-2358.
[13] P. Biswas, S. Pramanik, B.C. Giri, Aggregation of triangular fuzzy neutrosophic set information and its application to multiattribute decision making, Neutrosophic Set. Syst. 6 (2016), 20-40.

[14] J. Wang, G.W. Wei, M. Lu, An extended VIKOR method for multiple criteria group decision making with triangular fuzzy neutrosophic numbers, Symmetry 10 (2018), 497.

[15] J. Dombi, A general class of fuzzy operators, the demorgan class of fuzzy operators and fuzziness measures induced by fuzzy operators, Fuzzy Set. Syst. 8 (1982), 149-163.

[16] P.D. Liu, J.L. Liu, S.M. Chen, Some intuitionistic fuzzy Dombi Bonferroni mean operators and their application to multiattribute group decision making, J. Oper. Res. Soc. 69 (2017), $1-26$.

[17] J.Q. Chen, J. Ye, Some single-valued Neutrosophic Dombi weighted aggregation operators for multiple attribute decisionmaking, Symmetry, 9 (2017), 82.

[18] X.R. He, Typhoon disaster assessment based on Dombi hesitant fuzzy information aggregation operators, Nat. Hazard. 90 (2018), 1153-1175.

[19] R.R. Yager, Prioritized aggregation operators, Int. J. Approx. Reason. 48 (2008), 263-274.

[20] G.W. Wei, Hesitant fuzzy prioritized operators and their application to multiple attribute decision making, Knowl.-Based Syst. 31 (2012), 176-182.

[21] L.Y. Zhou, R. Lin, X.F. Zhao, G.W. Wei, Uncertain linguistic prioritized aggregation operators and their application to multiple attribute group decision making, Int. J. Uncertainty Fuzziness Knowl.-Based Syst. 21 (2013), 603-627.

[22] F.F. Jin, Z.W. Ni, H.Y. Chen, Interval-valued hesitant fuzzy Einstein prioritized aggregation operators and their applications to multi-attribute group decision making, Soft Comput. 20 (2016), 1863-1878.

[23] P.D. Liu, Y.M. Wang, Interval Neutrosophic prioritized OWA operator and its application to multiple attribute decision making, J. Syst. Sci. Complex. 29 (2016), 681-697.

[24] C. Bonferroni, Sulle medie multiple di potenze, Bollettino dell'Unione Matematica Italiana 5 (1950): 267-270. http://www. bdim.eu/item?id=BUMI_1950_3_5_3-4_267_0

[25] Z.S. Xu, R.R. Yager, Intuitionistic fuzzy bonferroni means, IEEE Trans. Syst. Man Cybern. B. 41 (2011), 568-578.

[26] W. Zhou, J.M. He, Intuitionistic fuzzy normalized weighted Bonferroni mean and its application in multicriteria decision making, J. Appl. Math. 2012 (2012), 1-22.

[27] Z.P. Tian, J. Wang, J.Q. Wang, et al., Multicriteria decision-making approach based on gray linguistic weighted Bonferroni mean operator, Int. Trans. Oper. Res. 15 (2018), 1635-1658.

[28] Q. Khan, P.D. Liu, T. Mahmood, F. Smarandache, K. Ullah, Some interval Neutrosophic Dombi power Bonferroni mean operators and their application in Multi-attribute decision making, Symmetry 10 (2018), 459.

[29] R.X. Nie, Z.P. Tian, J.Q. Wang, et al., Pythagorean fuzzy multiple criteria decision analysis based on Shapley fuzzy measures and partitioned normalized weighted Bonferroni mean operator, Int. J. Intell. Syst. 34 (2019), 297-324.

[30] Z.X. Li, H. Gao, and G.W. Wei, Methods for multiple attribute group decision making based on Intuitionistic Fuzzy Dombi Hamy mean operators, Symmetry 10 (2018), 574. 
[31] L.P. Wu, G.W. Wei, H. Gao, Y. Wei, Some interval-valued intuitionistic fuzzy Dombi Hamy mean operators and their application for evaluating the elderly tourism service quality in tourism destination, Mathematics 6 (2018), 294.

[32] C. Jana, T. Senapati, M. Pal, R.R. Yager, Picture fuzzy Dombi aggregation operators: application to MADM process, Appl. Soft Comput. J. 74 (2019), 99-109.

[33] H.R. Zhang, R.T. Zhang, H.Q. Huang, W. Jun, Some picture fuzzy Dombi Heronian mean operators with their application to Multiattribute decision making, Symmetry 10 (2018), 593.

[34] G.W. Wei, Z.P. Zhang, Some single-valued Neutrosophic Bonferroni power aggregation operators in multiple attribute decision making, J. Amb. Intel. Hum. Comp. 10 (2019), 863-882.

[35] P. Ji, J.Q. Wang, H.Y. Zhang, Frank prioritized Bonferroni mean operator with single-valued Neutrosophic sets and its application in selecting third-party logistics providers, Neural Comput. Appl. 30 (2016), 799-823.

[36] G. Noci, Designing 'Green' vendor rating systems for the assessment of a supplier's environmental performance, Eur. J. Purch. Supply Manag. 3 (1997), 103-114.

[37] A.H.I. Lee, H.Y. Kang, C.F. Hsu, et al., A green supplier selection model for high-tech industry, Expert Syst. Appl. 36 (2009), 7917-7927.
[38] Q.W. Cao, J. Wu, C.Y. Liang, An intuitionsitic fuzzy judgement matrix and TOPSIS integrated multi-criteria decision making method for green supplier selection, J. Intell. Fuzzy Syst. 28 (2015), 117-126.

[39] R.X. Liang, J.Q. Wang, H.Y. Zhang, A multi-criteria decisionmaking method based on single-valued trapezoidal neutrosophic preference relations with complete weight information, Neural Comput. Appl. 30 (2018), 3383-3398.

[40] J. Qin, X. Liu, W. Pedrycz, An extended TODIM multi-criteria group decision making method for green supplier selection in interval type-2 fuzzy environment, Eur. J. Oper. Res. 258 (2017), 626-638.

[41] M. Yazdani, P. Chatterjee, E.K. Zavadskas, et al., Integrated QFDMCDM framework for green supplier selection, J. Clean. Prod. 142 (2017), 3728-3740.

[42] J. Li, J.Q. Wang, An extended QUALIFLEX method under probability hesitant fuzzy environment for selecting green suppliers, Int. J. Fuzzy Syst. 19 (2017), 1866-1879.

[43] C. Ji, Z. Shouzhen, Z. Chonghui, An OWA distance-based, singlevalued neutrosophic linguistic TOPSIS approach for green supplier evaluation and selection in low-carbon supply chains, Int. J. Environ. Res. Public Health 15 (2018), 1439-1454. 